\title{
Prospective study of the role of HE4 and CA125 in treatment and follow-up in ovarian cancer patients
}

\section{Ann-Lisbeth Liest ( $\square$ lisbeth@liest.se )}

Linkopings universitet https://orcid.org/0000-0002-9515-1746

\section{Per Rosenberg}

Universitetssjukhuset i Linkoping

Ahmed Shaker Omran

Universitetssjukhuset i Linkoping

\section{Rasmus Mikiver}

Universitetssjukhuset i Linkoping

\section{Srinivas Uppugunduri}

Universitetssjukhuset i Linkoping

\section{Research article}

Keywords: Ovarian cancer, prognosis, HE4, CA125

Posted Date: April 6th, 2020

DOI: https://doi.org/10.21203/rs.3.rs-20697/v1

License: (c) (i) This work is licensed under a Creative Commons Attribution 4.0 International License. Read Full License

Version of Record: A version of this preprint was published at Obstetrics \& Gynecology International Journal on June 8th, 2020. See the published version at https://doi.org/10.15406/ogij.2020.11.00507. 


\section{Abstract}

Introduction: Epithelial Ovarian Cancer, tubal- and peritoneal cancer (EOC) is still the major cause of death in gynecological cancer. The outcome of primary surgery is an important prognostic factor. The primary aim of this study was to study the utility of HE4 and CA125 in monitoring the response of chemotherapy during treatment and in predicting prognosis and recurrence during follow-up. We have also evaluated the role of HE4 as a predictor of the result of surgical intervention.

Methods: Seventy-eight patients scheduled for chemotherapy were monitored with serum HE4 and CA125 during treatment and follow-up. In 39 patients samples for tumor markers were also obtained prior to surgical intervention.

Results: Both HE4 and CA125 decreased in response to treatment. PFS and OS were strongly dependent on HE4 levels prior to start of chemotherapy with significantly prolonged PFS and OS when HE4 levels were under upper reference limit of $82 \mathrm{pmol} / \mathrm{L}(\mathrm{P}=0.018$ resp. $P<0.001)$. The levels of HE4 correlated with primary surgical outcome with significantly lower postoperative HE4 in the radically operated group $(P<0.001)$. An increase in HE4 and/or CA125, signals a recurrence 3-6 months before diagnosis. Median levels for both HE4 and CA125 before start, during and at the end of treatment were significantly higher for platinum resistant patients $(P<0.005)$.

Conclusion: Both tumor markers are valuable in monitoring the response of chemotherapy as well as in predicting recurrence during follow-up. Postoperative HE4 holds promise as an objective marker to evaluate the result of surgery and is highly predictive for future prognosis.

\section{Introduction}

Epithelial Ovarian Cancer, tubal- and peritoneal cancer (EOC) is still the major cause of death in gynecological cancer despite improved chemotherapy and more recently introduction of new biological therapies. The outcome of primary surgery conducted with the intention to achieve complete cytoreduction is one of the main prognostic factors [1]. Patients presenting with a malignancy suspected to be originating from ovaries, fallopian tubes or peritoneum should be subjected either to primary debulking surgery or neoadjuvant chemotherapy (NACT) as initial treatment. However, since more than $60 \%$ are in advanced stage at diagnosis [2] complete primary cytoreduction is not always feasible. Neoadjuvant chemotherapy followed by delayed surgery has been proposed to result in the same clinical outcome as primary surgery in combination with post-operative chemotherapy when complete initial debulking is not deemed to be possible $[3,4]$. The decision to initiate debulking surgery is based on imaging diagnostics in combination with clinical and laboratory findings, performance status and comorbidity [3]. Serum levels of tumor marker CA125 are most often elevated, but have no established role in the decision regarding subsequent management. Delayed surgery, with the goal to achieve complete tumor debunking, should be considered after 3-4 cycles of NACT. In this context, a decrease of CA125 during NACT is an important factor. 
The tumor marker HE4 (Human Epididymis Protein) is used in the ROMA-algoritm (Risk of Ovarian Malignancy Algoritm) to distinguish a malignant pelvic mass from benign disorders [5]. HE4 has also been evaluated as a tool to predict the possiblity of achieving optimal surgical debulking, for monitoring the effect of chemotherapy and for predicting prognosis $[6,7,8,9,10,11]$.

The primary aim of this study was to study the utility of HE4 and CA125 in monitoring the response of chemotherapy during treatment as well as in predicting prognosis and recurrence during follow-up. Secondary aim was to evaluate the role of HE4 as a predictor of the result of surgical intervention.

\section{Materials And Methods}

This is a prospective, non-randomized multicenter study comprising patients from the referral area of the Department of Oncology, gynecologic section, University Hospital in Linköping, Sweden. The majority of the patients underwent surgery at the Department of Gynecology, University Hospital in Linköping, Sweden, but received chemotherapy at their referral hospitals in accordance with a treatment plan formulated by gynecologic oncologists at the University Hospital.

Women $\geq 18$ years of age diagnosed with ovarian cancer and planned for NACT or post-operative chemotherapy were included. Patients were recruited when they received information about the planned treatment. Informed consent was obtained from all patients. A total of 78 patients with EOC were included between November 2013 and April 2016. The first 39 patients participated in a study where samples for tumor markers were obtained prior to surgical intervention. The next 39 patients were first included after surgery or when NACT was initiated, which unfortunately resulted in a smaller number of participants with both pre- and postoperative marker values.

The patient group comprised predominantly of postmenopausal patients $(n=71)$ with median age 67 years. Only seven, median age 49 years were premenopausal. All patients were followed from start of chemotherapy up to 5 years, relapse or end of follow-up for other reasons. Blood samples for analysis of HE4 and CA125 were obtained at the days of chemotherapy. We also obtained samples within one week prior to surgery in 33 patients. Blood samples for analysis of tumor markers were also taken at each follow-up visit. The laboratory results were blinded for both clinicians and patients.

All patients were treated with Paclitaxel $175 \mathrm{~m}^{2}$ and Carboplatin AUC 5 combinations q3w aiming for 6 courses with the exception of three patients, who due to age and performance state received Carboplatin only. Reduction of doses, switch to other regimes as well as follow-up with 3-6 months interval was according to the national guidelines.

\section{Analysis}

All samples were transported at $-20^{\circ} \mathrm{C}$ and stored at $-80^{\circ} \mathrm{C}$ pending analysis. 
All CA125 and HE4 assays were performed at the Department of Clinical Chemistry, University Hospital, Linköping, Sweden to avoid inter-laboratory variability. Both CA125 and HE4 were measured by an electrochemiluminescent immunoassay (ECLIA) on the automated Cobas e602 (Roche Diagnostics GmbH, D68305 Mannheim, Germany). The CA125 method has been standardized against the Enzymun Test CA125 II method, which in turn has been standardized against the CA125 II RIA from Fujirebio Diagnostics. HE4 method has been standardized against the HE4 EIA method from Fujirebio Diagnostics, Inc. (Gothenburg, Sweden).

\section{Statistical analysis}

Difference between HE4- and CA125 expressions at different times and other variables were evaluated using Wilcoxon Signed Ranks Test and Mann-Whitney Test when appropriate. Survival curves were created using the Kaplan-Meier method, and statistical significance was determined by Log Rank (Mantel-Cox) test. For all statistical analysis, $P$ value of less than 0.05 was regarded as statistically significant. All statistical analyses were done with SPSS (version 25). The sample size was not sufficient to perform a multivariate analysis.

\section{Ethics}

The study was approved by the local committee of ethics. (Ref. 2012/141-31, 2013/424-31, 2014/330$32,2016 / 121 / 3,2018 / 64-32)$.

\section{Patient Characteristics}

Seventy-eight patients were included. One cohort of 39 patients was subjected to primary debulking surgery. Blood samples for analysis of tumor markers were obtained from 33 due to logistical mishaps in six cases. A second cohort of 39 patients had recorded tumor markers from the start of chemotherapy only. In this second group 23 patients underwent primary debulking surgery. Hence, in total, 62 patients underwent primary surgery. Complete debulking was achieved in 47 patients (75.8\%) out of these 62 patients. Sixteen patients commenced treatment with NACT followed by delayed surgical intervention in 12 cases. Complete cytoreduction was possible in only 4 of these (33.3\%). Four patients were not subjected to any surgical intervention at all.

The majority of patients 71 (91\%) were postmenopausal and seven (9\%) were premenopausal. All 27 patients diagnosed in early stages (FIGO I and II) were subjected to primary surgery, as were 35 of patients in advanced stages (FIGO III and IV). Sixteen patients with advanced disease started their treatment with chemotherapy. As expected the main histopathological types (86\%) were high-grade serous, clear cell and endometroid cancers. Low grade serous were found in $8 \%$ of the patients and $6 \%$ had serous non-graded cancers. 


\section{Results}

\section{Levels of HE4 and CA125}

In the cohort of 33 patients with recorded values of both pre- and postoperative tumor markers the median levels of HE4 significantly decreased to well under upper reference limit (URL) in the group in which complete debulking was achieved $(n=28)$ compared to the group $(n=5)$ left with minimal or bulky disease $(P<0.001$, Fig. $1 A-B)$. In the present study, we have used $82 \mathrm{pmol} / \mathrm{L}$ as the URL in accordance with conclusions from our previous study [12]. The four patients left with bulky disease had postoperative HE4 values, which were well above the URL. Similarly, patients with no macroscopic tumor postoperatively had significantly lower median postoperative CA125 values compared to those left with minimal or bulky disease $(P=0.002$, Fig. $1 \mathrm{C}-\mathrm{D})$. Eight patients $(22 \%)$ reported as radically operated had HE4 levels above URL postoperatively whereas 16 of 36 patients (44\%) still had CA125 levels above URL postoperatively. This difference persisted even when we combined both cohorts. In the whole material of 74 patients undergoing surgery 13 (17\%) had HE4 above URL and 28 (38\%) CA125 above URL when completely debulking surgery was reported.

There was no significant difference between median preoperative levels of neither HE4 nor CA125 in the groups with radical surgery versus non-radical surgery.

\section{Treatment}

In 6 out of 78 patients the planned sampling of tumor markers before the start of chemotherapy were not obtained due to logistical issues. Elevated HE4 levels decreased significantly between cycle 1 and 6 $(P<0.001)$. However normal HE4 levels at the start of chemotherapy did not decrease any further. Similarly CA125 levels above URL decreased significantly during treatment $(P<0.001)$.

\section{Progression free survival (PFS) and overall survival (OS)}

Progression free survival (PFS) was strongly dependent on the levels of HE4 prior to start of chemotherapy (Fig.2A). PFS was significantly prolonged if HE4 levels were below URL compared to patients with elevated HE4 levels at the start of treatment $(P=0.018)$. A pathologically elevated HE4 at cycle 1 indicated a shorter PFS even if the value normalized before cycle $6(P=0.012)$. Even OS is dependent on HE4 levels prior to start of chemotherapy with significantly prolonged OS $(P<0.001)$ in patients with HE4 values below URL. We also found that OS improved significantly $(P<0.05)$ even when HE4 values were above URL prior to start of chemotherapy but normalized at cycle 6 (Fig.2B). In these results patients achieving NACT are included. All these patients had high levels of both tumor markers. Likewise, a normal CA125 at cycle 1 indicated a significantly longer PFS compared to patients with elevated CA125 prior to chemotherapy ( $\mathrm{P}=0.017$, Fig.2C). However, neither PFS nor OS were significantly 
prolonged in patients with elevated CA125 levels at cycle 1 but normalized tumor marker at cycle 6 when compared to patients with CA125 above URL at the end of chemotherapy (Fig.2 C-D).

\section{Relapse}

The median follow-up was 35 months (range 0-68). Relapse was defined as CT verified signs of new tumor lesions in patients with no earlier evidence of disease. Both CA125 and HE4 most often reacted similarly with coinciding elevations in the 21 patients with relapse. An increase in HE4 levels preceded an increase in CA125 values in only two cases. The interval from first noticed increase of tumor markers above URL to a verified recurrence was between 2-6 months with a majority having a recurrence between 3-4 months (Fig.3).

\section{Platinum resistance}

In our material, 10 of 78 patients showed platinum resistance, defined as relapse less than 6 months after the last chemotherapy treatment or remaining tumor after platinum based chemotherapy. Median levels of both HE4 and CA125 before start, during and at the end of treatment were significantly higher $(P<0.005)$ in platinum resistant patients (Fig.4). No significant differences were observed median HE4 levels at the start of treatment, after three cycles and at the end of treatment in the platinum sensitive group. In patients with platinum resistant disease the median HE4 levels dropped during treatment from 651 to143 pmol/L after three cycles and to102 pmol/L at cycle 6 . All those patients had remaining elevation of CA125 at cycle 6 . In platinum sensitive patients the median CA125 level were over URL at the start of treatment but normalized after three cycles.

\section{Discussion}

Several authors have addressed the role of HE4 during treatment and follow-up. The preoperative level of HE4 has been advocated to be a predictor of the possibility of performing radical debulking surgery with reported cut-off values varying between $154-262 \mathrm{pmol} / \mathrm{L}[7,10,13,14]$. A preoperative level of HE4 above a median level of $394 \mathrm{pmol} / \mathrm{L}$ has been reported to be significantly associated with progression and mortality [15]. We could not corroborate these results as we found no significant differences in preoperative levels of neither HE4 nor CA125 in the group that was radically debulked compared to the group left with macroscopic disease. Our lack of corroboration could partly be due to limitations in our sample size with only a small number of patients with both pre- and postoperative tumor markers samples.

The result of the surgical intervention is assessed by the surgeon at the end of operation and currently there are no objective variables indicating radicality. It is therefore interesting to note that we found significantly lower median levels of HE4 and CA125 in patients where complete cytoreduction was achieved compared to the group left with minimal or bulky disease. Although CA125 also decreased after 
a successful cytoreduction, a majority of these patients still had elevated CA125 values. The levels of CA125 may be elevated postoperatively due to a peritoneal reaction after the surgical procedure. Hence, a postoperative elevation of CA125 has to be considered in relation to the time between surgery and blood sampling as well as the clinical condition of the patient. The median time from surgery to start of chemotherapy in our study was 42 days (range 13-92). It would therefore seem reasonable to conclude that surgical trauma has no impact on the CA125 levels prior to start of chemotherapy. However, as HE4 is reported to be independent of peritoneal reactions [16], and as the half time clearance is less than 4 hours [17], we would like to postulate that a normalized HE4 value postoperatively is a promising marker to evaluate the result of surgery and merits further investigation.

\section{Chemotherapy, PFS and OS}

The role of HE4 in monitoring chemotherapy has been evaluated regarding prognosis and prediction of platinum resistance. The serum levels of HE4 were under URL in $86 \%$ of patients reported as optimally tumor reduced. The median levels of HE4 in patients with normal values at the start of chemotherapy remained below URL during treatment whereas elevated HE4 levels at start of treatment decreased significantly. A similar result was seen for CA125 (Fig.5). Based on our data, we suggest that HE4 and CA125 are valid markers to monitor the response to chemotherapy, but only when the markers are above the normal range prior to start of chemotherapy. As a majority of patients (68\%) had elevated CA125 levels when treatment was started and only $42 \%$ had elevated HE4 levels, the role of CA125 as the most important marker for monitoring chemotherapy is thereby further confirmed and in line with Ferraro [18].

We found significantly prolonged PFS and OS in patients with HE4 levels under URL $82 \mathrm{pmol} / \mathrm{L}$ at the start of chemotherapy. Further, PFS was significantly shorter even when HE4 values were above the URL at start of chemotherapy but dropped to normal range after three and six cycles of chemotherapy. These results are in concordance with Steffensen et al. [6] who found a significant shorter PFS in 139 patients with high levels of HE4 just before start of chemotherapy. Also Kong et al. [9] described that the pretreatment HE4 level was a predictor of prognosis. On the other hand, in our study the level of CA125 was not predictive of either PFS or OS. Our results are in contrast to Rocconi et al. [19], who showed that both PFS and OS were significantly longer if CA125 was normalized after three cycles of chemotherapy regardless of the initial value.

\section{Relapse and Platinum resistance}

It is well known that the majority of patients treated for advanced EOC will have a relapse within few years after end of treatment. Definition of recurrence in the case of CA125 is as defined by the Gynecologic Cancer Intergroup [20].

The question about monitoring CA125 levels after adjuvant treatment for EOS in patients in complete remission has been intensively debated [21, 22, 23, 24]. Diagnosis of recurrent disease is often preceded 
by an increase of CA125, roughly two to six months before clinical symptoms, raising the possibility of starting chemotherapy based solely on increasing CA125 levels.

Rustin et al. [22] demonstrated no improved survival but decreased quality of life in a group with early retreatment compared to a group with start of treatment first after subjective symptoms. His study has been subject to criticism as the re-treatment regimens were very diverse and no patient underwent surgical intervention. On the other hand Fleming et al. [25] showed that delayed re-treatment, after an obvious increase in CA125 levels, decreased the possibility of achieving optimal tumor resection by a second surgical effort. To monitor and react on a rise in tumor markers could be further advocated by the DESKTOP III study that concluded that surgery in a situation of relapse affected prognosis but only when complete resection of all macroscopic tumor lesions could be attained and only in platinum-sensitive patients [26].

Reports concerning the role of HE4 during follow-up have suggested that HE4 is a more sensitive marker for diagnosis of recurrent EOC than CA125 [27, 28]. Most of these studies are small, but Steffensen et al. [11] demonstrated in a material of 88 patients that HE4 is a highly sensitive marker for relapsed disease. Anastasi et al. [29] observed an earlier increase of HE4 than CA125 in a very small group of eight patients with recurrence. In our material 21 recurrences were observed during the observation time of up to 68 months. Unfortunately, we did not succeed in obtaining samples from all planned visits as many dropped out due to practical difficulties. Despite this we can still conclude that both HE4 or CA125 alone or even better both together, indicate a recurrence with elevations occurring most often in parallel, roughly three to four months before confirmed recurrence (Fig. 5). Further research is needed to address the implications of increasing tumor markers.

It has been suggested that HE4 levels are a predictor of platinum resistance [30, 31].

Due to limited number of patients we have not investigated the role of HE4 in predicting the possibility of performing complete tumor resection after NACT. This is important for prognosis as a surgical intervention without complete tumor resection is of no benefit for the patient and in addition the interval to next chemotherapy might be prolonged. HE4 has been described as a useful tool in this question [27, 32].

In summary we can conclude that the levels of tumor markers at start of chemotherapy seem to be the most important factors for PFS and OS and also a predictor of platinum resistance. HE4 does not signal a recurrence earlier than CA125. We find the role of HE4 after surgical intervention to be of importance and suggest future studies of HE4 levels before and shortly after primary surgery for an objective evaluation of the surgical outcome and thereby also as a predictor of prognosis.

\section{Abbreviations}

HE4: Human Epididymal protein 4; CA125: Cancer Associated Antigen 125; PDS: Primary Debulking Surgery; NACT: NeoAdjuvant ChemoTherapy, PFS: Progression Free Survival; OS: Overall Survival; URL: 
Upper Reference Limit

\section{Declarations}

\section{Ethics}

This study was approved by the local committee of ethics: Regionala etikprövningsnämnden i Linköping, c/o Hälsouniversitetets kansli, Sandbäcksgatan 7, 58183 Linköping

Ref. 2012/141-31, 2013/424-31, 2014/330-32, 2016/121/3, 2018/64-32)

Consent for publication

Not applicable

Data available

The data files are available on request. Our data files are in Swedish language and Swedish numerical format (comma instead of full stop) and will be translated if needed.

Competing interests

The authors declare that they have no competing interests.

Funding

The authors gratefully acknowledge economic assistance from FORSS (Medical Research Council of Southeast Sweden), County Council of Ostergotland and the local fund Ostgotaregionens cancer fond. Both has contributed for costs of material for performing the analysis.

Authors contribution

Ann-Lisbeth Liest (ALL), Ahmed Omran (AO), Rasmus Mikiver (RM), Per Rosenberg (PR) and Srinivas Uppugunduri (SU) were all involved in different stages of this project. ALL, AO, PR and SU designed the study, formulated the study protocol and obtained permission from the local ethics board. ALL and PR recruited patients, $\mathrm{AO}$ was responsible for the clinical chemistry analyses and RM was responsible for performing the statistical analyses. All authors were involved in data analysis, data compilation and drafting preliminary and final versions of the manuscript.

All participants has read and approved the final manuscript.

Acknowledgements

We would like to thank Christina Jederud, responsible study-nurse for the project during initialization and first years of the study and Pernilla Nilsson, responsible study-nurse during the last years. Special thanks 
to all principal investigators, gynecologists and nurses from the recruiting centers in Linköping, Norrköping, Kalmar, Västervik, Jönköping, Värnamo, Eksjö, Nyköping and Västerås. Thanks also to SQRGC (Swedish Quality Registry of Gynecological Cancer).

Authors information

More information is presented in the Coverletter

\section{References}

1. du Bois A, Reuss A, Pujade-Lauraine E, Harter P, Ray-Coquard I, Pfisterer J. Role of surgical outcome as prognostic factor in advanced epithelial ovarian cancer: a combined exploratory analysis of 3 prospectively randomized phase 3 multicenter trials: by the Arbeitsgemeinschaft Gynaekologische Onkologie Studiengruppe Ovarialkarzinom (AGO-OVAR) and the Groupe d'Investigateurs Nationaux Pour les Etudes des Cancers de l'Ovaire (GINECO). Cancer. 2009;115:1234-44.

2. WHO. Globalcan. Assessed 2018-11-27.

3. Vergote I, du Bois A, Amant F, Heitz F, Leunen K, Harter P. Neoadjuvant chemotherapy in advanced ovarian cancer: On what do we agree and disagree? Gynecol Oncol. 2013;128:6-11.

4. Kehoe S, Hook J, Nankivell M, Jayson GC, Kitchener H, Lopes T et al. Primary chemotherapy versus primary surgery for newly diagnosed advanced ovarian cancer (CHORUS): an open-label, randomised, controlled, non-inferiority trial. Lancet. 2015;386:249-57.

5. Moore RG, Miller MC, Disilvestro P, Landrum LM, Gajewski W, Ball JJ, et al. Evaluation of the diagnostic accuracy of the risk of ovarian malignancy algorithm in women with a pelvic mass. Obstetrics and gynecology. 2011;118:280-8.

6. Steffensen KD, Waldstrom M, Brandslund I, Petzold M, Jakobsen A. The prognostic and predictive value of combined HE4 and CA-125 in ovarian cancer patients. International journal of gynecological cancer : official journal of the International Gynecological Cancer Society. 2012;22:1474-82.

7. Braicu El, Fotopoulou C, Van Gorp T, Richter R, Chekerov R, Hall C et al. Preoperative HE4 expression in plasma predicts surgical outcome in primary ovarian cancer patients: results from the OVCAD study. Gynecologic oncology. 2013;128:245-51.

8. Paek J, Lee SH, Yim GW, Lee M, Kim YJ, Nam EJ, et al. Prognostic significance of human epididymis protein 4 in epithelial ovarian cancer. Eur J Obstet Gynecol Reprod Biol. 2011;158:338-42.

9. Kong SY, Han MH, Yoo HJ, Hwang JH, Lim MC, Seo SS et al. Serum HE4 level is an independent prognostic factor in epithelial ovarian cancer. Ann Surg Oncol. 2012;19:1707-12.

10. Chudecka-Glaz AM, Cymbaluk-Ploska AA, Menkiszak JL, Sompolska-Rzechula AM, ToloczkoGrabarek Al, Rzepka-Gorska IA. Serum HE4, CA125, YKL-40, bcl-2, cathepsin-L and prediction optimal debulking surgery, response to chemotherapy in ovarian cancer. J Ovarian Res. 2014;7:62.

11. Steffensen KD, Waldstrom M, Brandslund I, Lund B, Sorensen SM, Petzold M et al. Identification of high-risk patients by human epididymis protein 4 levels during follow-up of ovarian cancer. Oncol 
Lett. 2016;11:3967-74.

12. Liest AL, Omran AS, Mikiver R, Rosenberg P, Uppugunduri S. RMI and ROMA are equally effective in discriminating between benign and malignant gynecological tumors: A prospective population-based study. Acta Obstet Gynecol Scand. 2019;98:24-33.

13. Shen Y, Li L. Serum HE4 superior to CA125 in predicting poorer surgical outcome of epithelial ovarian cancer. Tumour biology : the journal of the International Society for Oncodevelopmental Biology and Medicine. 2016;37:14765-72.

14. Angioli R, Plotti F, Capriglione S, Aloisi A, Montera R, Luvero D et al. Can the preoperative HE4 level predict optimal cytoreduction in patients with advanced ovarian carcinoma? Gynecologic oncology. 2013;128:579-83.

15. Trudel D, Tetu B, Gregoire J, Plante M, Renaud MC, Bachvarov D, et al. Human epididymis protein 4 (HE4) and ovarian cancer prognosis. Gynecologic oncology. 2012;127:511-5.

16. Galgano MT, Hampton GM, Frierson HF, Jr. Comprehensive analysis of HE4 expression in normal and malignant human tissues. Modern pathology : an official journal of the United States and Canadian Academy of Pathology, Inc. 2006;19:847-53.

17. Thompson C, Kamran W, Dockrell L, Khalid S, Kumari M, Ibrahim N et al. The Clearance of Serum Human Epididymis Protein 4 Following Primary Cytoreductive Surgery for Ovarian Carcinoma. Int J Gynecol Cancer. 2018;28:1066-72.

18. Ferraro S, Braga F, Lanzoni M, Boracchi P, Biganzoli EM, Panteghini M. Serum human epididymis protein 4 vs carbohydrate antigen 125 for ovarian cancer diagnosis: a systematic review. J Clin Pathol. 2013;66:273-81.

19. Rocconi RP, Matthews KS, Kemper MK, Hoskins KE, Huh WK, Straughn JM. The timing of normalization of CA-125 levels during primary chemotherapy is predictive of survival in patients with epithelial ovarian cancer. Gynecol Oncol. 2009;114:242-5.

20. Rustin GJ, Vergote I, Eisenhauer E, Pujade-Lauraine E, Quinn M, Thigpen T et al. Gynecological Cancer I. Definitions for response and progression in ovarian cancer clinical trials incorporating RECIST 1.1 and CA 125 agreed by the Gynecological Cancer Intergroup (GCIG). International journal of gynecological cancer : official journal of the International Gynecological Cancer Society. 2011;21:419-23.

21. Goonewardene TI, Hall MR, Rustin GJ. Management of asymptomatic patients on follow-up for ovarian cancer with rising CA-125 concentrations. The Lancet Oncology. 2007;8:813-21.

22. Rustin GJ, van der Burg ME, Griffin CL, Guthrie D, Lamont A, Jayson GC et al. Early versus delayed treatment of relapsed ovarian cancer (MRC OV05/EORTC 55955): a randomised trial. Lancet. 2010;376:1155-63.

23. Rustin GJ. Follow-up with CA125 after primary therapy of advanced ovarian cancer has major implications for treatment outcome and trial performances and should not be routinely performed. Annals of oncology : official journal of the European Society for Medical Oncology / ESMO. 2011;22 Suppl 8:viii45-viii8. 
24. Pignata S, Cannella L, Leopardo D, Bruni GS, Facchini G, Pisano C. Follow-up with CA125 after primary therapy of advanced ovarian cancer: in favor of continuing to prescribe CA125 during followup. Annals of oncology : official journal of the European Society for Medical Oncology / ESMO. 2011;22 Suppl 8:viii40-viii4.

25. Fleming ND, Cass I, Walsh CS, Karlan BY, Li AJ. CA125 surveillance increases optimal resectability at secondary cytoreductive surgery for recurrent epithelial ovarian cancer. Gynecol Oncol. 2011;121:249-52.

26. Bois An. ASCO 20192017. Available from: https://ascopubs.org >JC0.2017.35.15_suppl.5501. (Abstract)

27. Piovano E, Attamante L, Macchi C, Cavallero C, Romagnolo C, Maggino T et al. The role of HE4 in ovarian cancer follow-up: a review. International journal of gynecological cancer : official journal of the International Gynecological Cancer Society. 2014;24:1359-65.

28. Cao H, You D, Lan Z, Ye H, Hou M, Xi M. Prognostic value of serum and tissue HE4 expression in ovarian cancer: a systematic review with meta-analysis of 90 studies. Expert review of molecular diagnostics. 2018;18:371-83.

29. Anastasi E, Marchei GG, Viggiani V, Gennarini G, Frati L, Reale MG. HE4: a new potential early biomarker for the recurrence of ovarian cancer. Tumour Biol. 2010;31:113-9.

30. Chudecka-Glaz A, Cymbaluk-Ploska A, Wezowska M, Menkiszak J. Could HE4 level measurements during first-line chemotherapy predict response to treatment among ovarian cancer patients? PLoS One. 2018;13:e0194270.

31. Nassir M, Guan J, Luketina H, Siepmann T, Rohr I, Richter R et al. The role of HE4 for prediction of recurrence in epithelial ovarian cancer patients-results from the OVCAD study. Tumour biology : the journal of the International Society for Oncodevelopmental Biology and Medicine. 2016;37:3009-16.

32. Vallius T, Hynninen J, Auranen A, Carpen O, Matomaki J, Oksa S, et al. Serum HE4 and CA125 as predictors of response and outcome during neoadjuvant chemotherapy of advanced high-grade serous ovarian cancer. Tumour Biol. 2014;35:12389-95.

\section{Figures}




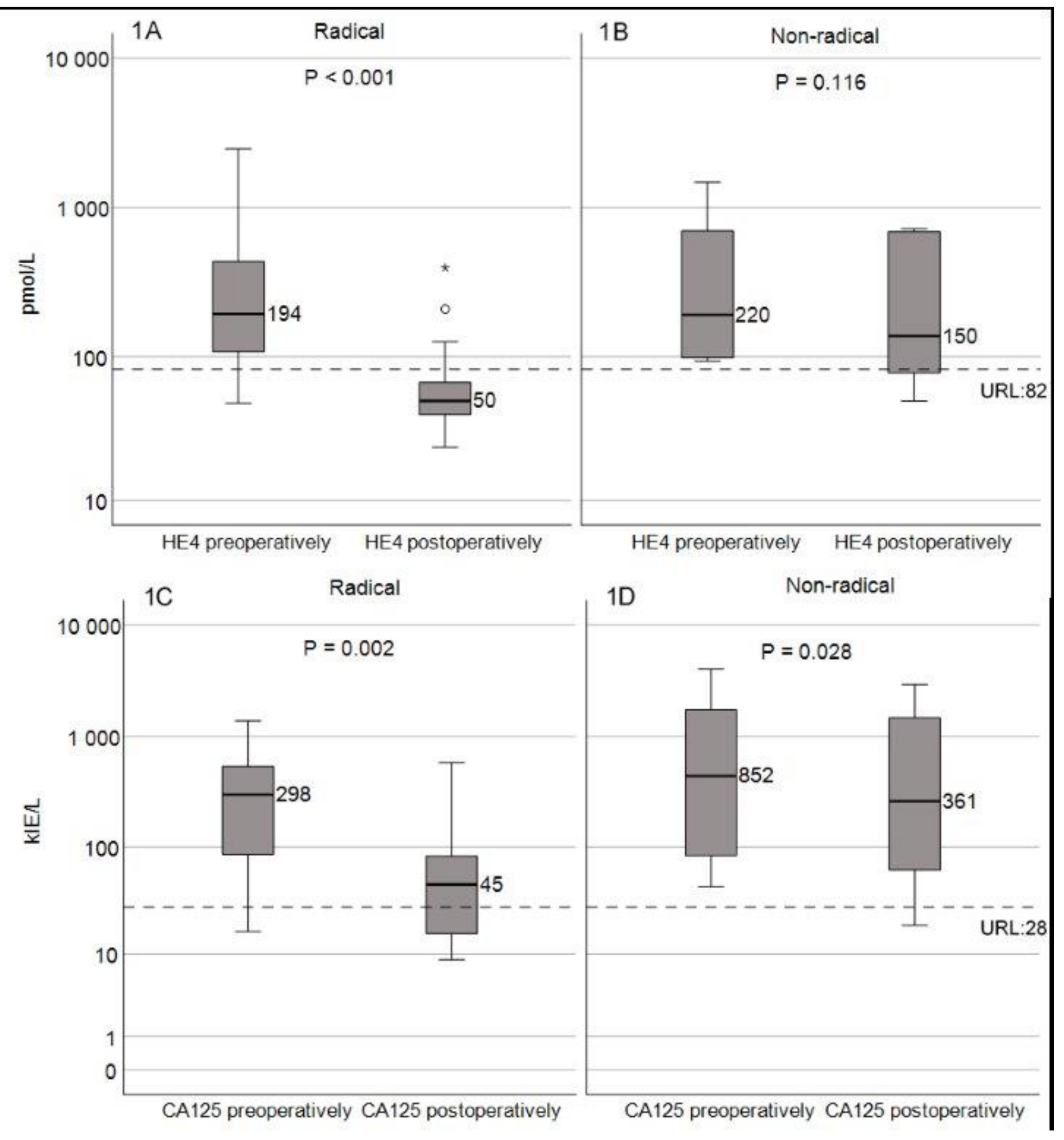

Figure 1

HE4 and CA125 and surgical outcome. HE4 and CA125 values related to surgical outcome 

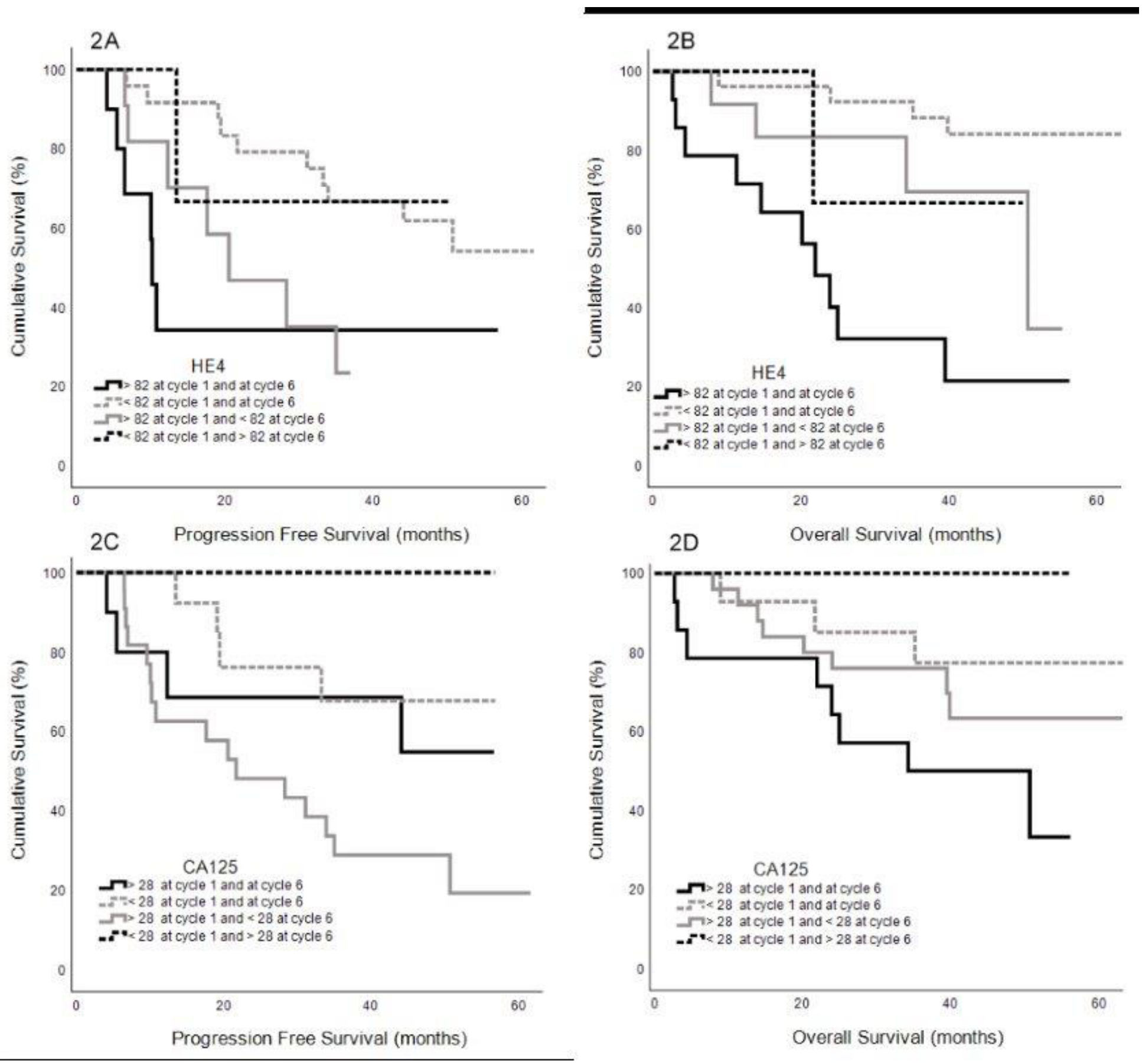

Figure 2

PFS and OS and marker levels. HE4 and CA125 levels related to PFS and OS 
Relapse
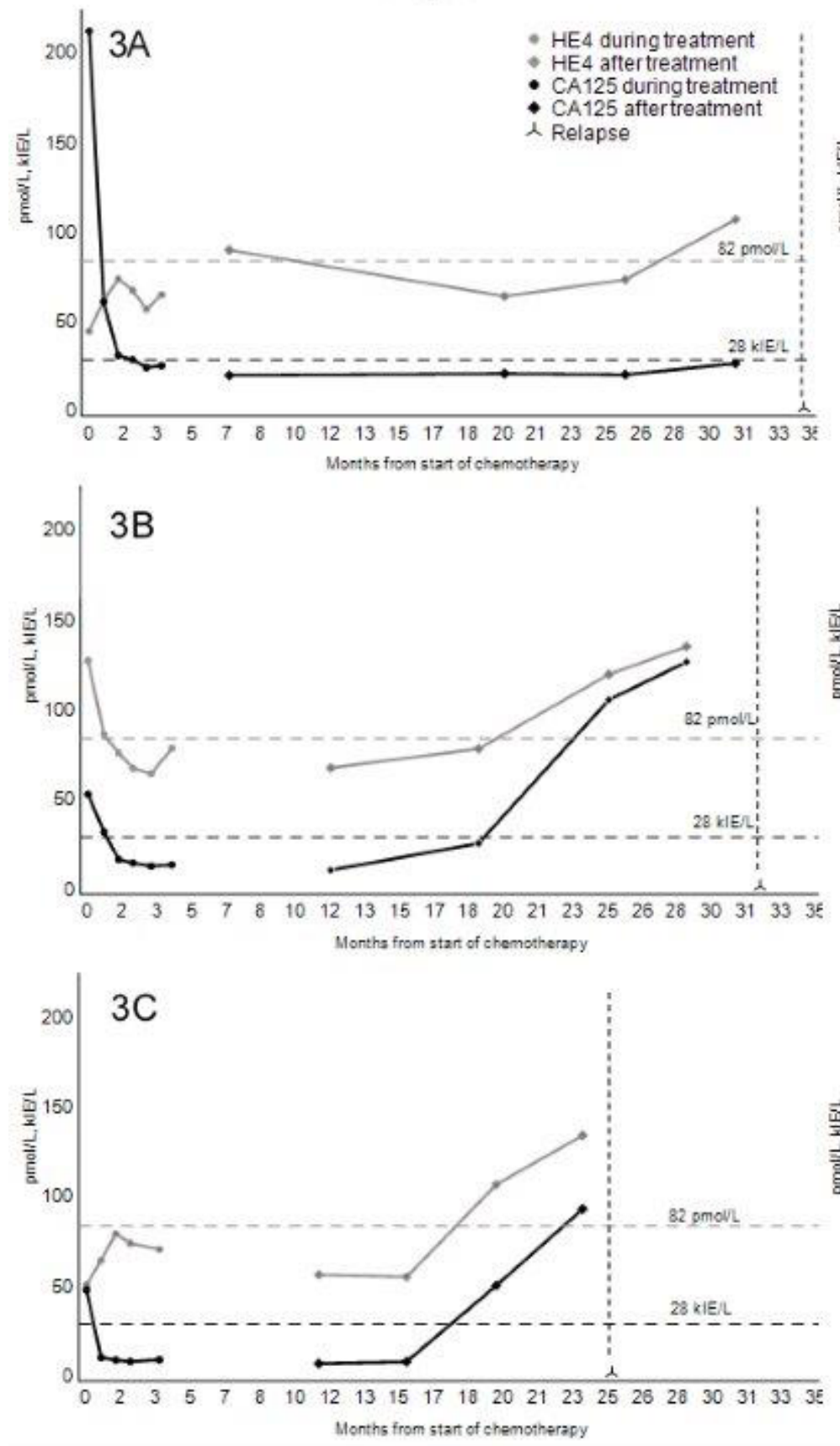

No evidence of disease (NED)
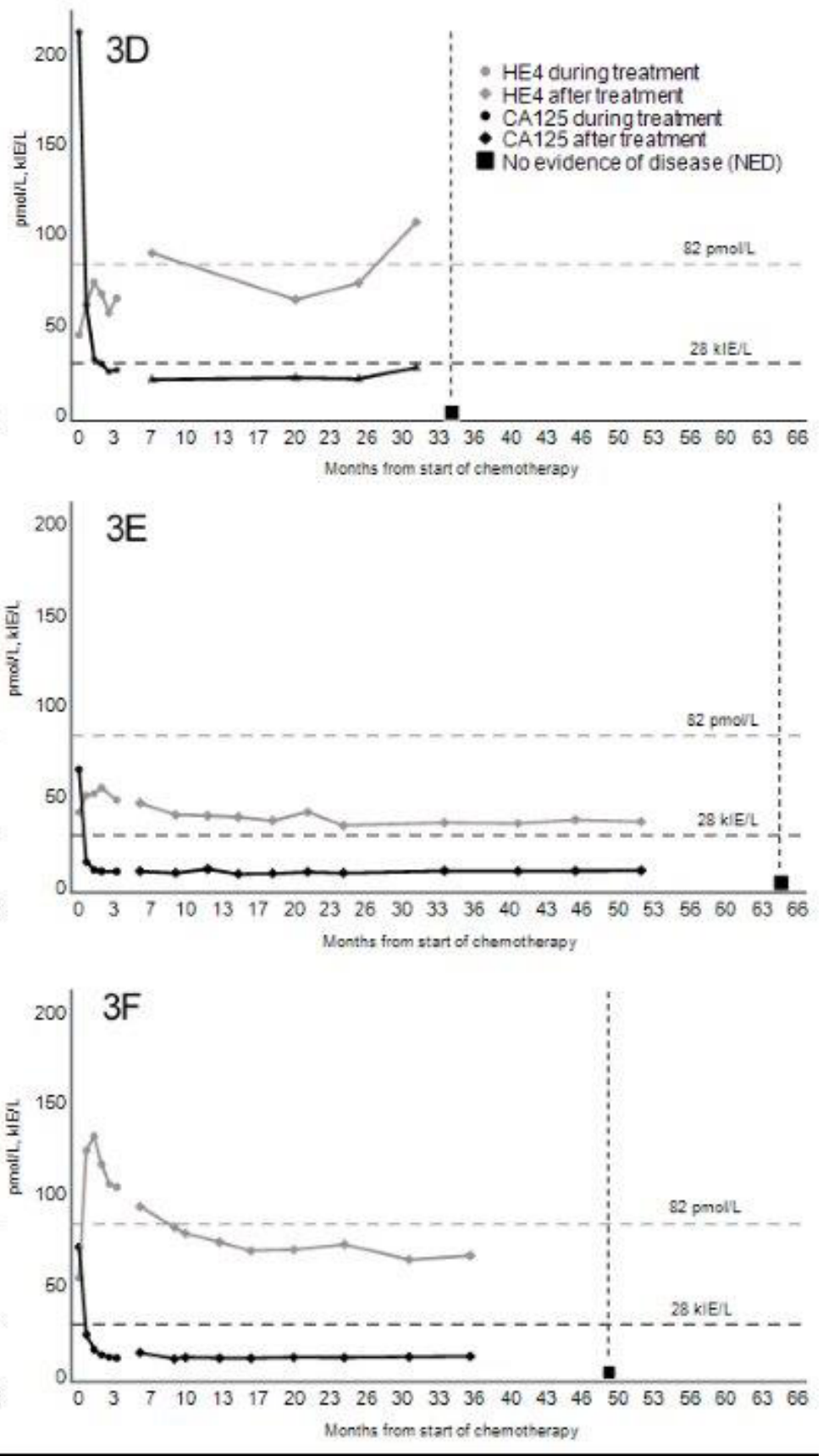

Figure 3

Relapse HE4 and CA125. HE4 and CA125 levels during follow-up in patients with and without relapse 

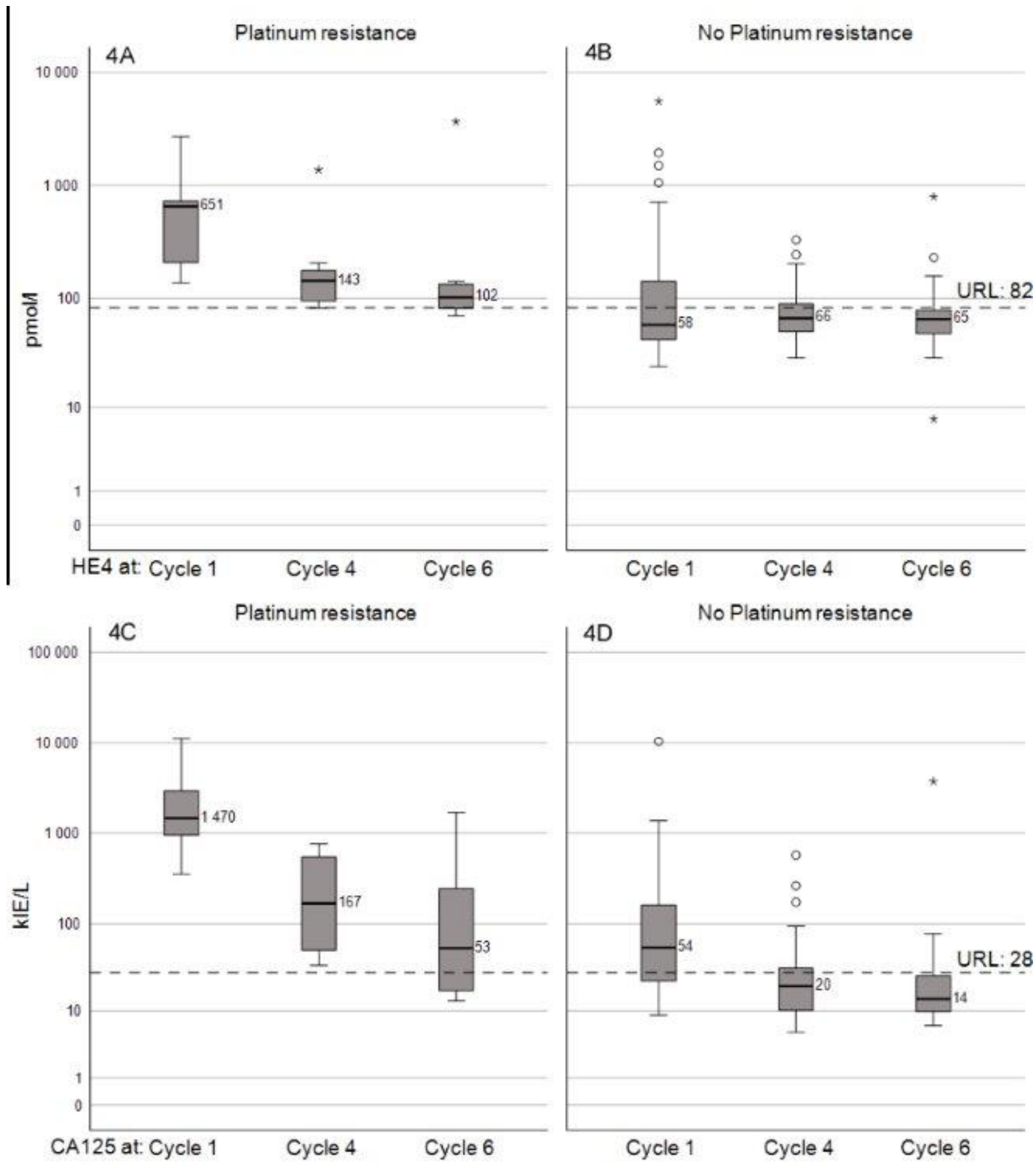

\section{Figure 4}

HE4 and CA125 levels related to platinum resistance. Tumor markers in patients that underwent all 6 cycles of chemotherapy in groups with platinum resistance (PR) and without platinum resistance (no PR). HE4 in total 52 patients (No PR $=43, P R=9)$. Ca12 2 in total 51 patients (No PR $=42, P R=9)$ 

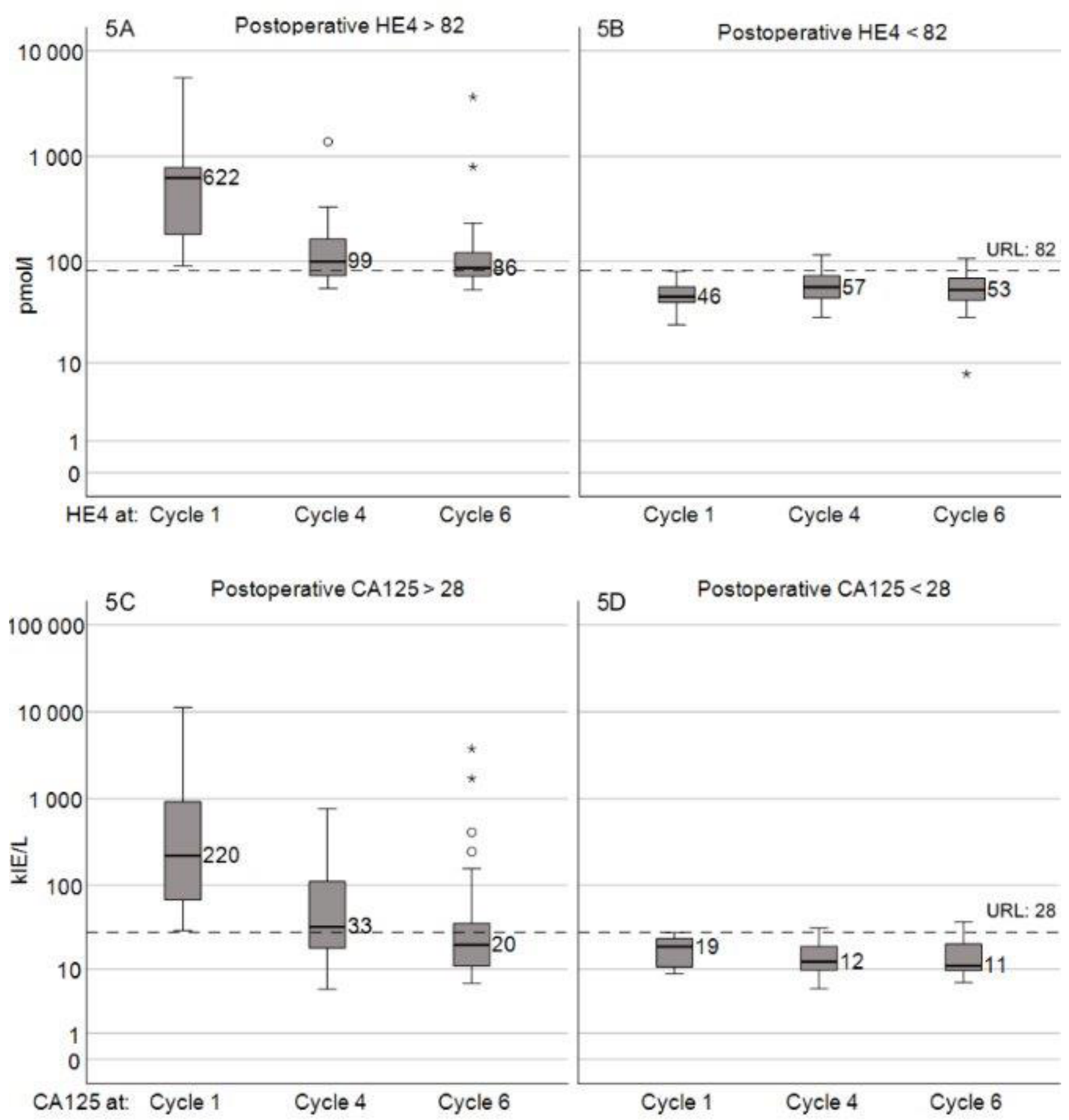

\section{Figure 5}

HE4 and CA125 during chemotherapy. Tumor markers during chemotherapy in patients that underwent all 6 cycles of chemotherapy. HE4 in total 54 patients (C1 >82: 24, C1<82: 28) CA125 in total 51 patients (C1 $>28: 36, \mathrm{C} 1<28: 15$ ) 